\title{
Diagnosis of Prodromal Alzheimer's Disease: Do You Really Want to Know?
}

Can. J. Neurol. Sci. 2010; 37: 2

The article by Porteri and colleagues ${ }^{1}$ in this issue of the CJNS is timely when considered in the context of the current emphasis on early detection/diagnosis of Alzheimer's disease (AD) and the attendant aim of arresting disease progression via the use of drugs modifying $\beta$-amyloid depositions in the brain. With clinical diagnostic criteria now established - impaired episodic memory and abnormal biological markers as reflected by MRI, PET, and/or $\mathrm{CSF}^{2}$ - a diagnosis of $\mathrm{AD}$ in its predementia stage may be possible, which would allow for novel trial designs and outcome measures such as "time to dementia," which compares a novel drug to placebo over a three year period.

Despite the apparent advantages of early detection, concerns have been raised regarding the risk of a catastrophic reaction ${ }^{3}$ in patients possessing the capacity to fully apprehend the meaning of a diagnosis of $\mathrm{AD}$, as well as the lack of data on the positive predictive value of the new diagnostic criteria and the elevated costs of the neuropsychological and biological evaluations compared to the usual dementia work-up. The current publication ${ }^{1}$ illustrates the impact of the new diagnostic criteria on two participants enrolled in a prospective cohort study. In addition to demonstrating a sensitive approach to the issue of disclosure the authors highlight the importance of planning $a$ priori for the disclosure of incidental findings in prospective cohort studies (such as silent tumors on brain imaging), and for positive findings with diagnostic implications, as was observed to the case with the two aforementioned volunteers. Protocols, ethics review and consent forms should anticipate these issues.

The ethics of research in the area of $\mathrm{AD}$, where competency to consent is the main issue, must now be broadened in order to incorporate both those persons at high risk of $\mathrm{AD}$ as well as those with a diagnosis of very early $\mathrm{AD}$, who have yet to be attained by dementia. The REVEAL Study ${ }^{4}$ offers a somewhat reassuring perspective in that a differential reaction subsequent to disclosure of carrier status was not found between those at higher risk of developing $\mathrm{AD}$ due their status as an apolipoprotein E4 carriers and those with the usual age-associated risk. Nevertheless the "catastrophic reaction scale" used prospectively in the REVEAL study could be administered prior to the disclosure of the diagnosis of pre-dementia AD.

Another issue that needs revisiting is the reluctance of many research ethics committees and investigators to allow persons with mild AD to choose whether they wish to try a new drug as opposed to placebo. The currently available symptomatic drugs for $\mathrm{AD}$ (cholinesterase inhibitors and the NMDA-receptor antagonist memantine) may be considered "standard drug treatment" by the Canadian Consensus Conference on the Diagnosis and Treatment of Dementia ${ }^{5}$, yet their effect size is a far cry from the benefits observed with levodopa in Parkinson's disease. There is no valid reason why competent patients should not be allowed to decide for themselves whether to delay the commencement of symptomatic drugs, the benefits of which are limited, in order to try new drugs of potentially greater therapeutic effect. In the case of persons diagnosed with predementia AD, the placebo-controlled studies could extend as long as dementia is not yet present. It is time to ask the patients what they want, rather than telling them what is best for them.

Serge Gauthier, Antoine Leuzy McGill Center for Studies in Aging Douglas Mental Health University Institute Montreal, Quebec, Canada

\section{REFERENCES}

1. Porteri C, Galluzzi S, Geroldi C, Frisoni GB. Diagnosis disclosure of prodromal Alzheimer disease - ethical analysis of two cases. Can J Neurol Sci. 2010; 37: 67-75.

2. Dubois B, Feldman HH, Jacova C, Dekosky ST, Barberger-Gateau $\mathrm{P}$, Cummings $\mathrm{J}$, et al. Research criteria for the diagnosis of Alzheimer's disease: revisiting of the NINCDS-ADRDA criteria. Lancet Neurol. 2007; 6: 734-46.

3. Gauthier S, Sheltens P. Can we do better in developing new drugs for Alzheimer's disease? Alzheimers Dement. 2009; 5: 489-91.

4. Green RC, Roberts JS, Cupples LA, Relkin NR, Whitehouse PJ, Brown T, et al. Disclosure of APOE genotype for risk of Alzheimer's disease. N Engl J Med. 2009; 361: 245-54.

5. Hogan DB, Bailey P, Carswell A, Clarke B, Cohen C, Forbes D, et al. Management of mild to moderate Alzheimer's disease and dementia. Alzheimers Dement. 2007; 3: 355-84. 